\title{
Higher Ultrasonic Frequency Liquid Phase Exfoliation Leads to Larger and Monolayer to Few-Layer Flakes of 2D Layered Materials
}

Madina Telkhozhayeva ${ }^{a}$, Eti Teblum ${ }^{a}$, Rajashree Konar $^{a}$, Olga Girshevitz ${ }^{b}$, Ilana Perelshtein $^{b}$, Hagit Aviv ${ }^{a}$, Yaakov R. Tischler ${ }^{a}$, Gilbert Daniel Nessim $^{a} *$

a The Department of Chemistry and Institute of Nanotechnology, Bar-Ilan University, Ramat Gan 52900, Israel.

b Institute of Nanotechnology \& Advanced Materials, Bar-Ilan University, Ramat Gan 52900, Israel.

* Corresponding author.

E-mail address: Gilbert.Nessim@,biu.ac.il (G.D. Nessim).

Tel: +972-52-717-4255; +97237384540

I Authors contributed equally to this work. 


\section{Description of the dropcasting process}

Dropcasting is considered a facile, low-cost, and fast deposition technique. It has been shown that the proper solvent (surface tension), drop volume, substrate, and drying-up process are the key factors that affect the physical properties (shape, thickness, and size) of the deposited flakes. ${ }^{1,2}$ Here, the dropcasting method was controlled by using a small volume $(\sim 1 \mathrm{~mL})$ of a precursor solution with low surface tension.

However, it should be noted that the exfoliation method, rather than the deposition technique, affects the measured lateral size of the flakes. Additionally, the flake size distribution can be controlled by post-treatment methods, e.g., centrifugation. It was reported that the average lateral flake size decreases for increased centrifugation rates (rpm). ${ }^{3}$ In our case, we did not apply such post-sonication methods. 

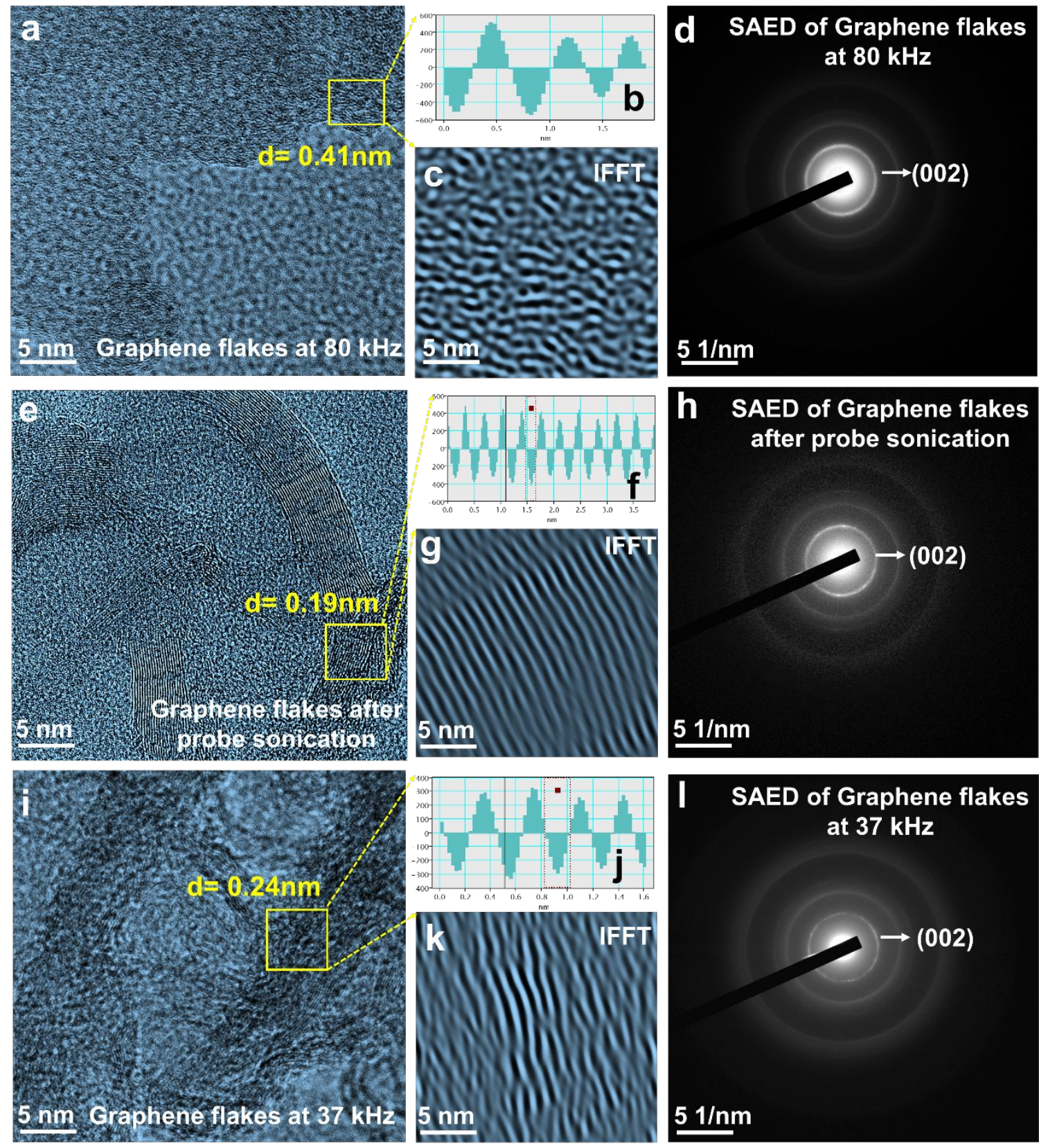

$51 / \mathrm{nm}$
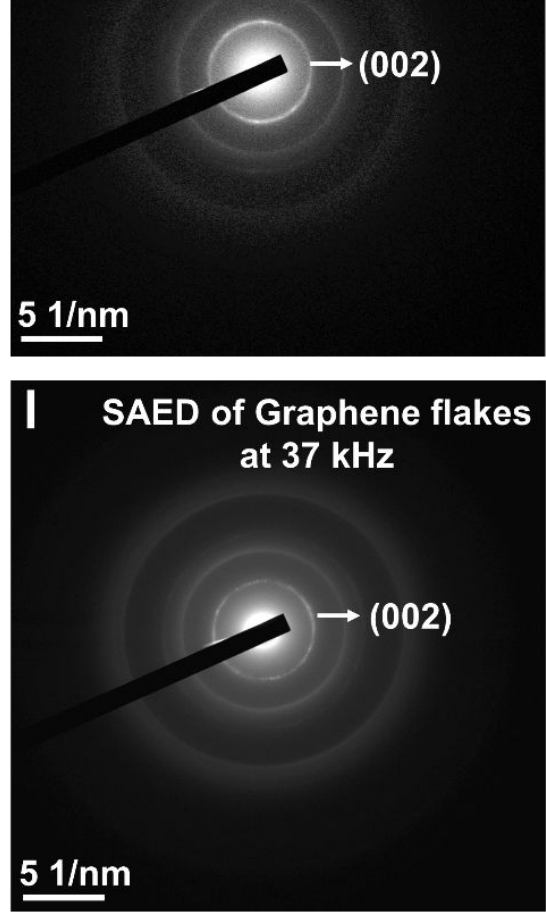

Figure S1. (a) High-Resolution Transmission Electron Microscopy (HRTEM) of Graphene flakes at 80 kHz with the corresponding Inverse Fast Fourier Transform (IFFT) depicted in (b) and (c) and (d) Selected Area Diffraction of Graphene flakes at $80 \mathrm{kHz}$; (e) High-Resolution Transmission Electron Microscopy (HRTEM) of Graphene flakes after probe sonication with the corresponding Inverse Fast Fourier Transform (IFFT) depicted in (f) and (g) and (h) Selected Area Diffraction of Graphene flakes after probe sonication; (i) High-Resolution Transmission Electron Microscopy (HRTEM) of Graphene flakes at $37 \mathrm{kHz}$ with the corresponding Inverse Fast Fourier Transform (IFFT) depicted in (j) and (k) and (l) Selected Area Diffraction of Graphene flakes at $37 \mathrm{kHz}$. 


\section{Raman spectroscopy}

Figure S2 compares the representative Raman spectra of graphene exfoliated at $80 \mathrm{kHz}$ bath, 37 $\mathrm{kHz}$ bath, and probe sonication. Typically, the $\mathrm{D}$ band indicates the presence of defects, and the intensity ratio between the $\mathrm{D}$ and $\mathrm{G}$ bands $\left(\mathrm{I}_{\mathrm{D}} / \mathrm{I}_{\mathrm{G}}\right)$ quantifies the defects in graphene layers. ${ }^{4} \mathrm{We}$ observed a weak disorder-related D band $\left(\sim 1344 \mathrm{~cm}^{-1}\right)$ in the spectrum of graphene exfoliated at $80 \mathrm{kHz}$ bath. The $\mathrm{I}_{\mathrm{D}} / \mathrm{I}_{\mathrm{G}}$ ratio for the ethanol-dispersed graphene at $80 \mathrm{kHz}$ bath was $\sim 0.17$, which is much lower compared to graphene obtained using direct liquid exfoliation of graphite with the addition of salts or surfactants. ${ }^{5,6}$ Our result supports the HRTEM observations, showing that the sonication process at higher frequency did not result in the formation of significant quantities of defects on the graphitic basal plane. Observations of very narrow G-peak can also confirm the high-quality of exfoliated flakes. Basal plane defects typically occur in graphene oxide (GO) or reduced $\mathrm{GO}(\mathrm{rGO})$ and lead to the broadening of the $\mathrm{G}$ band. ${ }^{7}$ In our case, the $\mathrm{G}$ band is narrow, indicating that high-frequency sonication-assisted exfoliation led to few basal plane defects. For solvent exfoliated graphene flakes, the defects are generally attributed to the edge effects which are formed as a result of cutting primarily large crystallites into smaller flakes during sonication. Although the values of $\mathrm{I}_{\mathrm{D}} / \mathrm{I}_{\mathrm{G}}$ for graphene exfoliated at $37 \mathrm{kHz}$ bath and for probe sonication are low, the intensity ratio $\mathrm{I}_{\mathrm{G}} / \mathrm{I}_{2 \mathrm{D}}$ increased with the number of graphene layers $(1.00,2.44$, and 2.07 for graphene exfoliated at $80 \mathrm{kHz}$ bath, $37 \mathrm{kHz}$ bath, and probe sonication, respectively). Additionally, the shape of the 2D band (peak intensity and width) can be affected by the number of layers, ${ }^{8}$ which may help to understand why we measured a $\mathrm{I}_{\mathrm{G}} / \mathrm{I}_{2 \mathrm{D}}$ ratio of 1.00 for the $80 \mathrm{kHz}$ bath, higher than what we would have anticipated given that our extensive statistical AFM analysis showed that $77 \%$ of our flakes were less than three-layer thick. 
a)

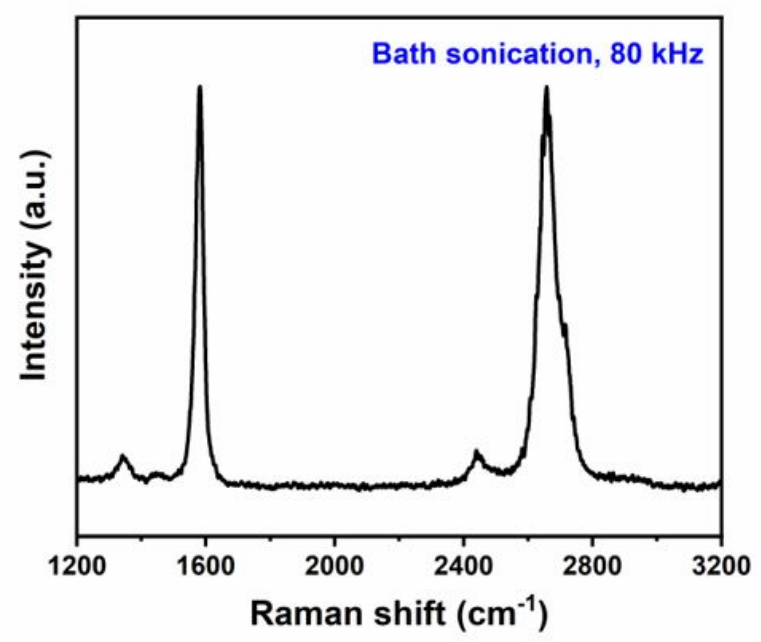

b)

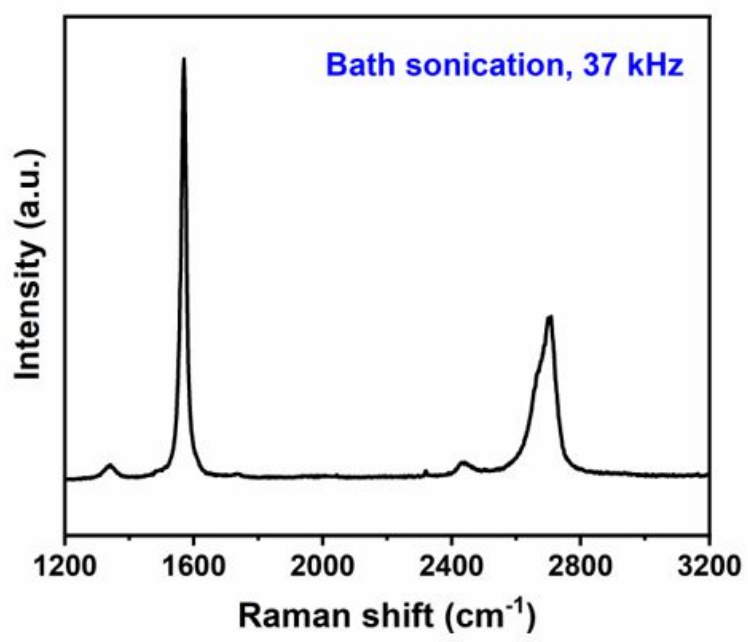

c)

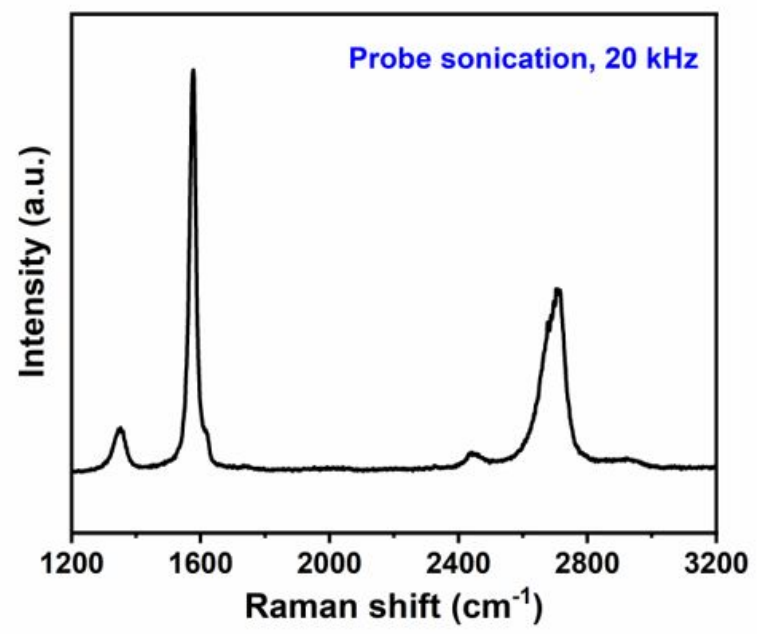

Figure S2. Raman spectra of the exfoliated graphene by applying (a) $80 \mathrm{kHz}$ frequency (100 W); (b) 37 $\mathrm{kHz}$ frequency $(100 \mathrm{~W})$, and (c) probe sonication $(20 \mathrm{kHz}$ frequency, $750 \mathrm{~W})$. 


\section{REFERENCES}

(1) He, P.; Derby, B. Controlling Coffee Ring Formation during Drying of Inkjet Printed 2D Inks. Adv. Mater. Interfaces 2017, 4 (22), 2-7. https://doi.org/10.1002/admi.201700944.

(2) Eslamian, M.; Soltani-Kordshuli, F. Development of Multiple-Droplet Drop-Casting Method for the Fabrication of Coatings and Thin Solid Films. J. Coatings Technol. Res. 2018, 15 (2), 271280. https://doi.org/10.1007/s11998-017-9975-9.

(3) Khan, U.; O’Neill, A.; Porwal, H.; May, P.; Nawaz, K.; Coleman, J. N. Size Selection of Dispersed, Exfoliated Graphene Flakes by Controlled Centrifugation. Carbon N. Y. 2012, 50 (2), 470-475. https://doi.org/10.1016/j.carbon.2011.09.001.

(4) Pimenta, M. A.; Dresselhaus, G.; Dresselhaus, M. S.; Cançado, L. G.; Jorio, A.; Saito, R. Studying Disorder in Graphite-Based Systems by Raman Spectroscopy. Phys. Chem. Chem. Phys. 2007, 9 (11), 1276-1291. https://doi.org/10.1039/b613962k.

(5) Niu, L.; Li, M.; Tao, X.; Xie, Z.; Zhou, X.; Raju, A. P. A.; Young, R. J.; Zheng, Z. Salt-Assisted Direct Exfoliation of Graphite into High-Quality, Large-Size, Few-Layer Graphene Sheets. Nanoscale 2013, 5 (16), 7202-7208. https://doi.org/10.1039/c3nr02173d.

(6) Guardia, L.; Fernández-Merino, M. J.; Paredes, J. I.; Solís-Fernández, P.; Villar-Rodil, S.; Martínez-Alonso, A.; Tascón, J. M. D. High-Throughput Production of Pristine Graphene in an Aqueous Dispersion Assisted by Non-Ionic Surfactants. Carbon N. Y. 2011, 49 (5), 1653-1662. https://doi.org/10.1016/j.carbon.2010.12.049.

(7) Ferrari, A. C.; Meyer, J. C.; Scardaci, V.; Casiraghi, C.; Lazzeri, M.; Mauri, F.; Piscanec, S.; Jiang, D.; Novoselov, K. S.; Roth, S.; Geim, A. K. Raman Spectrum of Graphene and Graphene Layers. Phys. Rev. Lett. 2006, 97 (18), 1-4. https://doi.org/10.1103/PhysRevLett.97.187401.

(8) Das, A.; Chakraborty, B.; Sood, A. K. Raman Spectroscopy of Graphene on Different Substrates and Influence of Defects. Bull. Mater. Sci. 2008, 31 (3), 579-584. https://doi.org/10.1007/s12034008-0090-5. 